\title{
INFLUENCE OF METAL VAPOURS ON RADIATION CHARACTERISTICS OF AIR ARC PLASMAS
}

\author{
M. Bartlová*, P. Kloc, V. Aubrecht, N. Bogatyreva \\ Faculty of Electrical Engineering and Communicatin, Brno University of Technology, Technická 10, 61600 \\ Brno, Czech Republic \\ * bartlova@feec.vutbr.cz
}

\begin{abstract}
This paper deals with the evaluation of radiation properties of air arc plasma with various admixtures of $\mathrm{Cu}, \mathrm{Ag}$, and $\mathrm{Fe}$, respectively. Under assumption of isothermal plasma cylinder, the net emission coefficients were calculated for various arc radii as a function of the plasma temperature up to $30000 \mathrm{~K}$. For plasma with prescribed temperature profile, the equation of radiation transfer was solved in the $\mathrm{P}_{1}$ approximation, and the radiation flux and its divergence were calculated.
\end{abstract}

Keywords: radiation transfer, net emission coefficient, $\mathrm{P}_{1}$ approximation.

\section{Introduction}

Thermal plasma is widely used in various technological applications: welding, cutting, spraying, switching processes in relays, commutators or circuit breakers, etc. The electric arc is created between two electrodes typically made from copper, silver or carbon materials and their compositions with high-melting components (tungsten, molybdenum, chromium, etc.). Process efficiency is defined by different parameters that determine arc operation (temperature, pressure, concentrations of species, size of plasma) and which influence the energy transfer within the medium (conduction, convection, radiation, Joule effect). All these energy exchanges depend on the medium, which is modified by the presence of the arc and by the appearance of new species from contact erosion. The influence of these materials and their erosion are the key factors for a better understanding of the physical phenomena occurred in plasma and effects on the energy transfer process. The nature of the materials, used to make the electrodes, is important in optimizing processes, since their degradation leads to the modification of their surface and to the change of the parameters of plasma. In the past, many theoretical works focused on the properties of thermal plasma in the presence of metallic vapors $[1,2]$. Even small admixture significantly alter the plasma properties such as radiation and thermal conductivity. The experimental investigations were done mostly using optical emission spectroscopy techniques, which allows to determine the plasma temperature and density, the electrodes surface temperature, and many other arc properties, and the effects of different arc conditions [3]. Recent achievements of numerical modelling of arc processes allow to investigate self-consistently the cathode and anode, the electrode sheaths, and the arc column regions [4]. The radiation arc properties, predicted theoretically on the base of fundamental databases, are often in good agreement with experimental measurements.
In this paper we present the calculation of radiation characteristics for mixtures of air thermal plasma and vapours of copper, silver and iron. The choice of mixtures was guided by usual applications (transferred arc, arc welding, plasma spraying of metallic particles).

\section{Absorption of radiation}

Absorptivity $\kappa_{\nu}$ is proportional to the concentrations of the chemical species occurring in the plasma. We assume that arc plasma is in the state of the local thermodynamic equilibrium (LTE) and its composition can be computed as an equilibrium composition by, for example, minimizing the Gibbs energy of the system [5]. We have taken into account diatomic molecules, atoms and up to triple ions of N, O, C, Ar and metal elements.

Both line and continuum radiation were considered in calculation of absorption coefficients. Two types of transition constitute the continuous spectrum: the bound-free transition (photoionization - a photon is absorbed by an atom or ion and the electron is ejected and goes into one of the continuum free energy states) and free-free transition (bremsstrahlung - a photon is absorbed by a free electron that makes a transition to another free state). The calculation of the absorption coefficients due to the photoionization of the excited atoms depends on the corresponding photoionization cross sections given by Opacity Project [6]. For the high levels of atoms and ions, the latter are obtained according to the hydrogen-like approximation [7]. The hydrogen-like approximation has been used also for calculation of free-free transitions.

The second part of the database deals with boundbound transitions for a specific wavelength between two bound energy levels of the atom or ion and forming the spectrum of lines. Most of this study is to build a complete database comprising, for each transition, the properties of higher and lower levels, the transition probability, the associated oscillator strength 


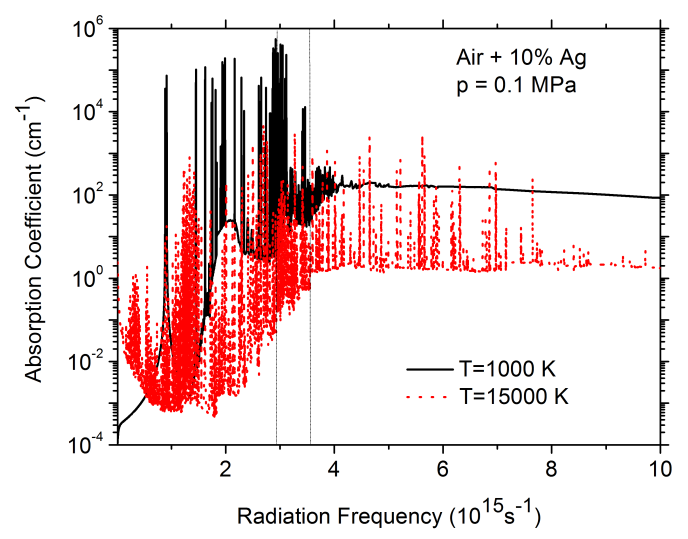

Figure 1. Absorption coefficient of air plasma with admixture of 10 vol\% $\mathrm{Ag}$ at temperatures of $1000 \mathrm{~K}$ and $15000 \mathrm{~K}$ and positions of optimal band boundaries determined from (6).

and the parameters of Doppler, Stark, Van der Waals, resonance and natural spectral line broadening. Semiempirical formulas given in [7] were used for calculation of various broadening mechanisms. The tabulated values of oscillator strengths were taken from the NIST database [8] for atoms and ions of N, O, C, Ar and Fe, and from Kurucz's database [9] for $\mathrm{Cu}$ and $\mathrm{Ag}$. The line shapes in our calculations are given by Voigt profile. The multiplet structure and the lines overlapping have been considered.

At low temperatures, we need to study the complexity of the molecular spectrum that we can break down into two parts: the first part formed of a continuous molecular background whose emission is mainly due to bound-free transitions corresponding to photodissociation and photoionization, the second represented by molecular bands superimposed on the continuous background resulting from the transitions between the discrete electronic levels of the molecule. Molecular photodissociation and photoionization cross sections were taken from PHIDRATES database [10], for calculation of molecular band absorption the Franck-Condon principle [11] was used. The calculated total radiation absorption coefficients as a function of frequency for plasma temperature of $1000 \mathrm{~K}$ and $15000 \mathrm{~K}$ are plotted for the plasma systems of air $+10 \mathrm{vol} \% \mathrm{Ag}$ and air $+10 \mathrm{vol} \% \mathrm{Fe}$ in figures 1 and 2, respectively. As can be seen, the absorption spectrum is very complex and widely varying function of frequency.

\section{Net emission coefficient}

The calculation of the radiative transfer taken into account in most hydrodynamic models requires the description of energy exchanges by radiation at each point of the mesh. Two major difficulties arise when the radiative transfer equation needs to be coupled to conservation equations of mass, energy, momentum. Indeed, the radiative transfer equation must in principle be solved for each of the wavelengths and in each

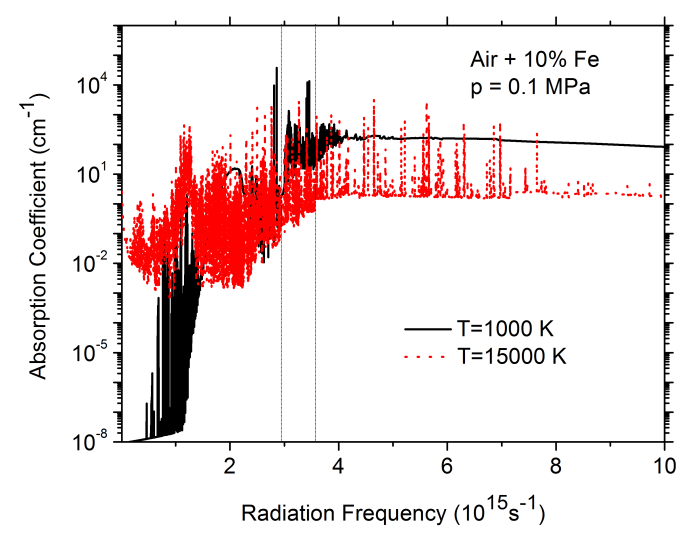

Figure 2. Absorption coefficient of air plasma with admixture of 10 vol\% Fe at temperatures of $1000 \mathrm{~K}$ and $15000 \mathrm{~K}$ and positions of optimal band boundaries determined from (6).

of the directions, which represents a colossal computation time. Some simplifications are then considered, which can be of two types: a spectral simplification of the radiation and a geometric or spatial simplification of radiation propagation within the structure.

One of the methods for spatial simplification is the net emission coefficient (NEC) approximation, originally proposed by Lowke [12]. The total emission coefficient of a plasma reflects the total power emitted per unit volume of a plasma. Net emission is a similar quantity taking into account the self-absorption of radiation. NEC determines the value of the power radiated locally by the volume element from which is subtracted the power emitted by the rest of the plasma and absorbed by the volume element. Under certain conditions (an isothermal, homogeneous, spherical plasma), the net emission coefficient $\varepsilon_{\mathrm{N}}$ then corresponds to the divergence of the radiative flux $\mathbf{F}_{R}$ (net emission of radiation), which is an essential term of energy loss in the energy conservation equation used in arc models

$$
\nabla \cdot \mathbf{F}_{\mathrm{R}}=4 \pi \varepsilon_{\mathrm{N}}
$$

The net emission coefficient $\varepsilon_{N}$ at the center of the sphere depends on the plasma composition, the pressure of the medium $p$, its temperature $T$ and the radius of the plasma $R_{\mathrm{p}}$

$$
\varepsilon_{\mathrm{N}}=\int_{0}^{\infty} B_{\nu}(T) \kappa_{\nu}(T, p) \mathrm{e}^{-\kappa_{\nu}(T, p) R_{\mathrm{p}}} \mathrm{d} \nu
$$

with $B_{\nu}$ the black body radiation, $\kappa_{\nu}$ the monochromatic absorption coefficient corrected of the induced emission, $R_{\mathrm{p}}$ the radius of the sphere or cylinder considered (the results for the isothermal sphere are approximately the same as for the isothermal cylinder). The exponential term reflects the self-absorption of radiation. The radius $R_{\mathrm{p}}$ is determined to be the maximum plasma radius for which the medium can 


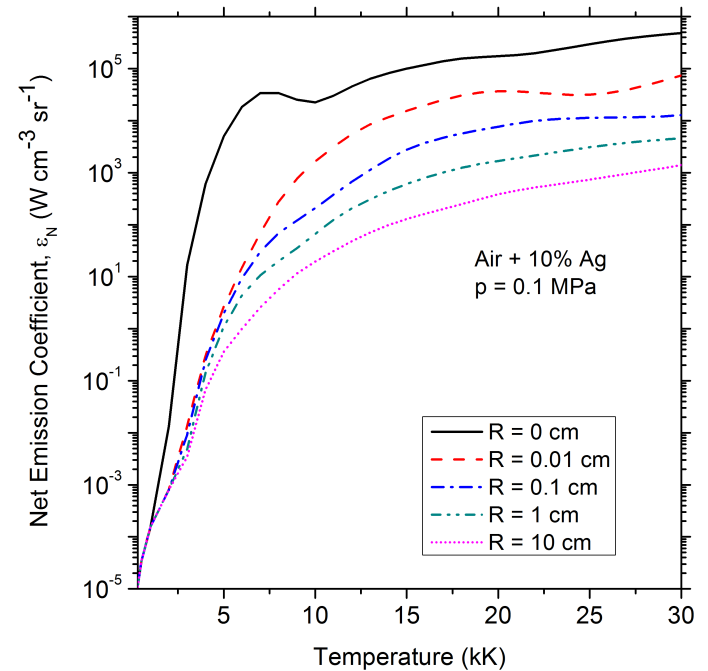

Figure 3. Net emission coefficient of air plasma with 10 vol\% Ag for various plasma radii $R$.

be considered isothermal. This typically corresponds to the characteristics of the radial temperature profile of an arc plasma that can be described by a slow temperature decrease in the central part and then a rapid drop in temperature at the edge.

Despite seemingly very restrictive assumptions, this type of coefficient is mostly used in the modeling of arcs and thermal plasmas for three reasons: extreme simplification of radiation calculation in models; good approximation of the net power radiated by the hottest regions of the plasma in which the temperature gradients are relatively low; taking into account the frequency dependence of the radiation.

All calculations were performed at atmospheric pressure for temperatures between $300 \mathrm{~K}$ and $30000 \mathrm{~K}$. Examples of calculated net emission coefficients for various radii of a plasma cylinder of air $+10 \mathrm{vol} \% \mathrm{Ag}$ and air +10 vol\% Fe are given in figures 3 and 4 , respectively. Depending on the temperature, the net emission coefficient rises very rapidly below a limit temperature (about $10000 \mathrm{~K}$ ) from which the latter progresses more slowly. It can be explained by the presence of ionic lines above $10000 \mathrm{~K}$ which increase the total radiation. Then, we notice a decrease of the net emission coefficient in relation with the increase of the radius of the plasma highlighting the phenomenon of absorption. Note that the case $R_{\mathrm{p}}=0 \mathrm{~cm}$ corresponds to the fictitious case of an optically thin plasma for which absorption is considered as zero.

Figure 5 shows the influence of iron metal vapour on the net emission coefficient of air plasma. For an atmospheric pressure, the results of the radiation are presented for the plasma radius of $0.1 \mathrm{~cm}$ in function of temperature for several volume proportions of iron $(0 \%, 1 \%, 5 \%, 10 \%)$. We can see that even a small proportion of iron significantly increases net radiation at

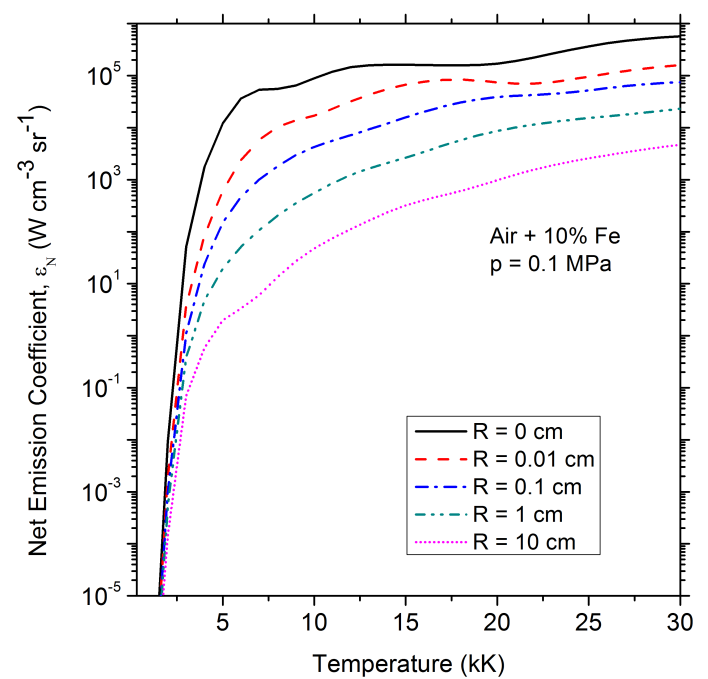

Figure 4. Net emission coefficient of air plasma with 10 vol\% Fe for various plasma radii $R$.

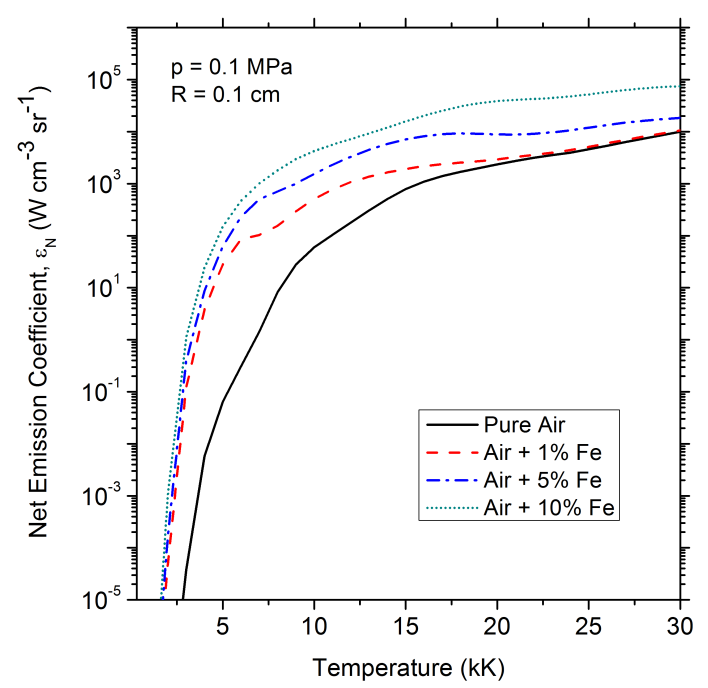

Figure 5. Net emission coefficients for three composition variants of 1,5 and 10 vol\% $\mathrm{Fe}(R=0.1 \mathrm{~cm})$.

low temperatures. This phenomenon is explained by the rapid ionization of iron whose ionization potential is lower than that of other considered species. The ions thus created and the associated transitions then cause a sharp increase in the net emission coefficient. Figure 6 shows the effect of various metals $(\mathrm{Cu}, \mathrm{Ag}$, $\mathrm{Fe}$ ) in proportion of $10 \mathrm{vol} \%$ for the plasma radius of $0.1 \mathrm{~cm}$. The greater influence of iron is due to the richness of the iron spectrum whose many lines are little absorbed. Comparison of our values of net emission coefficients of air +1 vol\% Ag with those of [1] at the pressure of $0.1 \mathrm{MPa}$ for plasma radius of $0.1 \mathrm{~cm}$ is presented in figure 7 . Difference in the results can be 


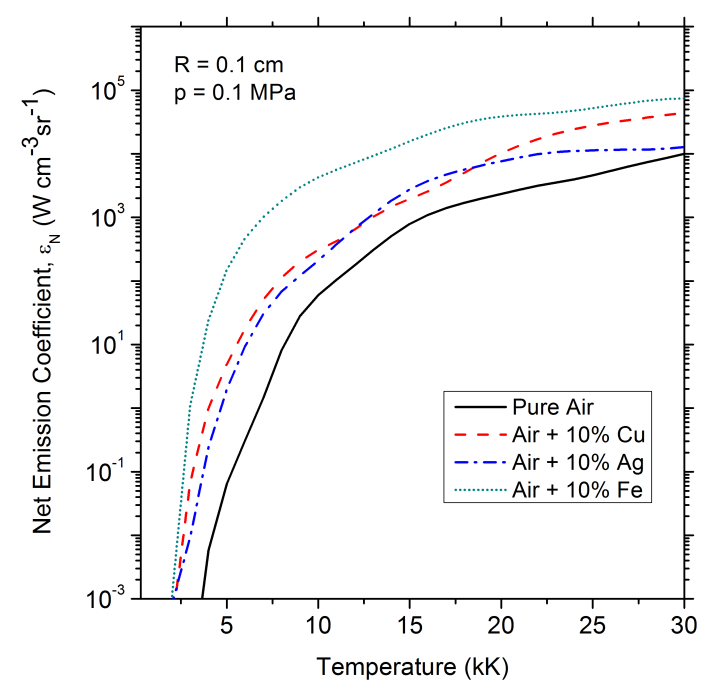

Figure 6. Net emission coefficients for 10 vol\% admixtures of $\mathrm{Ag}, \mathrm{Cu}$ and $\mathrm{Fe}(R=0.1 \mathrm{~cm})$.

explained by different treatment of spectral line overlapping and by different input spectral data. Spectral lines in [1] are treated using escape factor, the line overlapping is neglected. In our work, we use line by line integration, respecting the overlapping. 764 lines for Ag with data of oscillator strengths from Kurucz's database [9] were considered in our work, only 24 lines for $\mathrm{Ag}$ were taken into account in [1], probably from NIST database [8].

\section{4. $P_{1}$ approximation and mean absorption coefficients}

The method of NEC is very simple to use since it does not require the resolution of any additional equation. In fact, for a given temperature and a given plasma radius, it gives a coefficient of volume losses which has been calculated and tabulated beforehand. It is widely used in the modeling of thermal plasmas and makes possible to take into account in the energy conservation equation radiation losses of the hot parts of the plasma. It thus remains a very good approximation if one is not interested in the radiative phenomena of the plasma but only in its hydrodynamic aspect. However, when one wants to evaluate the radiative transfer in the arc and take into account the radiation absorption of the hot parts by the colder parts at the edges, it is necessary to use more complex methods. All radiation models are based on the radiative transfer equation. This differential equation represents the conservation of radiation for a volume element. Under the assumption of local thermodynamic equilibrium and neglecting the scattering, the equation of radiation transfer can be written in the form

$$
\boldsymbol{\Omega} \cdot \nabla I_{\nu}(\mathbf{r}, \boldsymbol{\Omega})=\kappa_{\nu}\left(B_{\nu}-I_{\nu}(\mathbf{r}, \boldsymbol{\Omega})\right)
$$

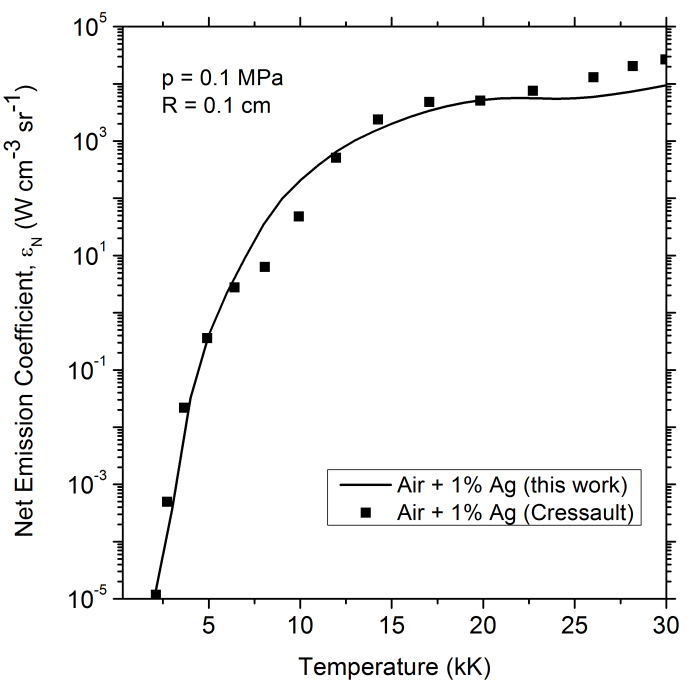

Figure 7. Comparison of calculated net emission coefficients with [1].

where $I_{\nu}$ is the spectral intensity of radiation, $\Omega$ is a unit direction vector, $B_{\nu}$ is the Planck black-body intensity. The resolution of this differential equation is complicated because it is a differential equation with variable coefficients that are functions of the wavelength, the local temperature, the direction considered and the composition of the medium. Various methods have been used to calculate the radiation of thermal plasmas. We can mention among them the method of partial characteristics [13], the discrete ordinate (SN) method [14] and finally the $P_{1}$ method [15]. Each of these methods uses simplifying assumptions about the spectral dependence of equation (3) or spatial dependence and often both. In this paper, we have chosen to use the $\mathrm{P}_{1}$ model. This model seems to give satisfactory results on the radiation emitted by the hot parts of the plasma and takes into account the absorption of this radiation by the colder parts.

The $\mathrm{P}_{1}$ model comes from a more general family of models that are the $\mathrm{P}_{\mathrm{N}}$ models. These models simplify the radiative transfer equation (3) by developing the radiative intensity $I_{\nu}$ in the form of a series of spherical harmonics. In $\mathrm{P}_{1}$ approximation, the spherical series development is truncated to order 1. Putting $I_{\nu}$ in this form in (3), multiplying the equation (3) by spherical harmonics, integrating over all directions and exploiting the orthogonality properties of spherical harmonics, the equation of radiative transfer (3) becomes a differential equation with unknown incident radiation $G_{\nu}$

$$
\nabla \cdot\left(-\frac{1}{3 \kappa_{\nu}} \nabla G_{\nu}(\mathbf{r})\right)+\kappa_{\nu} G_{\nu}(\mathbf{r})=4 \pi \kappa_{\nu} B_{\nu}
$$

where $-\frac{1}{3 \kappa_{\nu}} \nabla G_{\nu}(\mathbf{r})=\mathbf{F}_{\nu}(\mathbf{r})$ is the radiation flux. The associated Marshak boundary condition at a 
black-body diffuse wall take the form

$$
\mathbf{n} \cdot \nabla G_{\nu}=\frac{3}{2} \kappa_{\nu}\left(4 \pi B_{\nu}^{\text {wall }}-G_{\nu}\right)
$$

where $\mathbf{n}$ is the local outward surface normal.

For spectral simplification, we assume that the plasma radiates as a "gray body" across the spectrum or in bands, and therefore the absorption coefficient no longer varies with frequency. The "mean absorption coefficient" method (MAC) consists of cutting the spectrum into different previously defined and justified bands in which the absorption coefficient will be considered constant for a given temperature. The limits of the frequency bands depend on the considered plasma system. The definition of the bands must satisfy both the accuracy of the results and the limitation of the computation cost. However, there is no clear guideline how to choose the frequency limits. One way how to find the optimal band selection is using of numerical optimization. The band selection based on this approach leads to the three-band model [16] with cutting frequencies (see Figs. 1 and 2)

$$
(0.001,2.938,3.513,10) \times 10^{15} \mathrm{~Hz}
$$

Even though the frequency limits were found by numerical optimization they correspond also to some important features in the absorption coefficients, namely to the ionization of the nitrogen atom from the basic and the first excited level. In order to eliminate the overestimated influence of strong spectral lines on the value of averaged absorption coefficient, the $k$ distribution type model [15] was used: the frequencies within each band (6) were further divided into four sub-bands according to the value of $\kappa_{\nu}\left(\right.$ in $\left.\mathrm{cm}^{-1}\right)$

$$
\kappa_{\nu} \in[0,0.1],[0.1,1],[1,10],[10, \infty] .
$$

Two methods for the calculation of the mean absorption coefficients are defined in the literature [17]: the Planck MAC $\bar{\kappa}_{\mathrm{P}}$ (defined on the assumption of optically thin, emission dominated system) and Rosseland MAC $\bar{\kappa}_{\mathrm{R}}$ (developed for optically thick, absorption dominated system)

$$
\begin{gathered}
\bar{\kappa}_{\mathrm{P}}=\frac{\int_{\Delta \nu} \kappa_{\nu} B_{\nu} \mathrm{d} \nu}{\int_{\Delta \nu} B_{\nu} \mathrm{d} \nu} . \\
\bar{\kappa}_{\mathrm{R}}^{-1}=\frac{\int_{\Delta \nu} \kappa_{\nu}^{-1} \frac{\mathrm{d} B_{\nu}}{\mathrm{d} T} \mathrm{~d} \nu}{\int_{\Delta \nu} \frac{\mathrm{d} B_{\nu}}{\mathrm{d} T} \mathrm{~d} \nu},
\end{gathered}
$$

Using MAC in equation (4), the net emission of radiation is

$$
\nabla \cdot \mathbf{F}_{\mathrm{R}}=\sum_{j} \nabla \cdot \mathbf{F}_{j}
$$

where $j$ denotes the individual frequency bands.

The radiative transfer inside the cylindrical plasma column at atmospheric pressure with prescribed temperature profile [16] was calculated using the $\mathrm{P}_{1}$ approximation. The plasma system consisted of air with

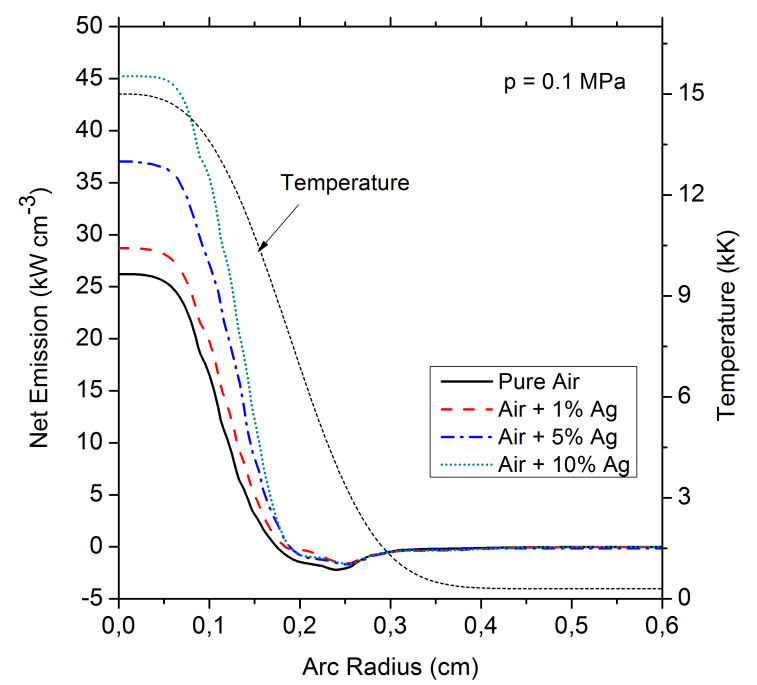

Figure 8. Net emission as a function of radial distance for pure air and 1, 5 and 10 vol\% $\mathrm{Ag}$.

various admixtures of $\mathrm{Cu}, \mathrm{Ag}$ and $\mathrm{Fe}$. While respecting the conditions under which Planck and Rosseland MAC are defined, a combination of their values was used - Planck MAC was used in frequency bands with low values of absorption coefficients, Rosseland MAC in frequency bands with strong absorption, i.e. in subbands with $\kappa>1 \mathrm{~cm}^{-1}$. It was shown in [18] for pure air plasma that this approximation gives relatively good accuracy when compared to the exact spectral integration. Figs. 8 and 9 show the effect of silver and iron vapours on the net emission of air plasma, respectively. Metal admixtures contribute mainly to the radiation emission in the hot central region. The influence of metal vapours increases with increasing admixture ratios. Metals are more emissive than air due to their electronic structure and rich line spectrum. On the other hand, the absorption of radiation in the cold regions at the edge of the arc is almost independent of the metal concentration when this concentration remains low (up to $10 \%$ ). Our results are similar to those of $[14,19]$. It should be noted that the results for silver are probably underestimated. Due to the lack of data we did not take into account $\mathrm{Ag}^{2+}$ and $\mathrm{Ag}^{3+}$ ions.

\section{Conclusions}

Calculation of radiation characteristics of air thermal plasma with various admixtures of copper, silver and iron have been performed. Under assumption of an isothermal plasma, net emission coefficients were calculated at the atmospheric pressure for temperatures up to $30000 \mathrm{~K}$. Our results for Ag admixtures were compared with data available in the literature. Our data and the literature data deviate slightly due to the different treatment of spectral lines and different input spectral data. 


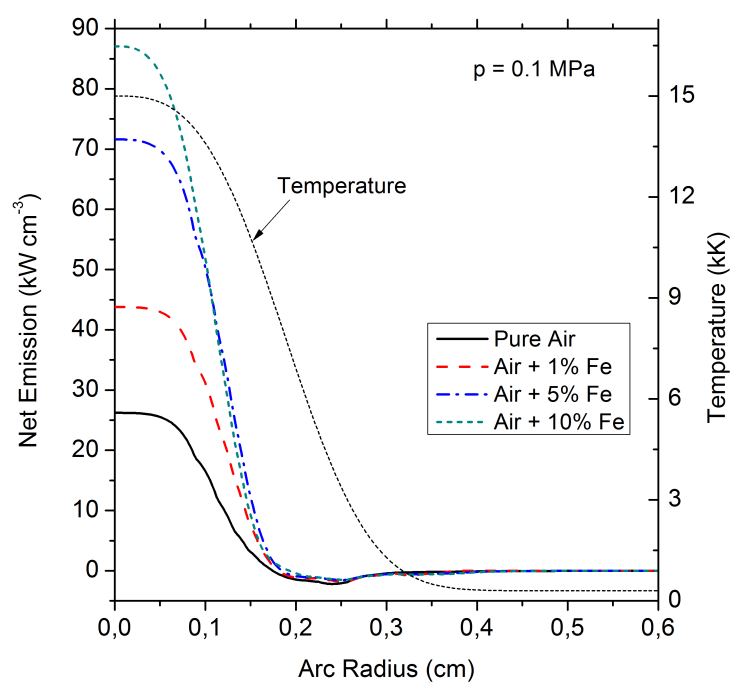

Figure 9. Net emission as a function of radial distance for pure air and 1, 5 and 10 vol\% $\mathrm{Fe}$.

To consider the reabsorption of radiation in the outer and cooler parts of the arc plasma, the $\mathrm{P}_{1}$ approximation was used to calculate the net emission (divergence of radiation flux) in the cylindrical plasma column at atmospheric pressure with prescribed temperature profile. Spectral simplification was provided using the $k$-distribution type model with the combination of Planck and Rosseland mean absorption coefficients. Due to their higher emissivity, metals mainly increase radiation losses. The effect of metal admixture on radiation absorption is much smaller, especially for small amounts of admixtures (up to $10 \%)$.

\section{Acknowledgements}

This research work was carried out in the Centre for Research and Utilization of Renewable Energy (CVVOZE). The authors gratefully acknowledge financial support from the Ministry of Education, Youth and Sports of the Czech Republic under NPU I programme (project No. LO1210 Energy for Sustainable Development) and OP VVV Programme (project No. CZ.02.1.01/0.0/0.0/16 $13 / 0001638$ CVVOZE Power Laboratories-Modernization of Research Infrastructure).

\section{References}

[1] Y. Cressault et al. Influence of metallic vapours on the properties of air thermal plasmas. Plasma Sources Sci. Technol., 17(3):035016, 2008. doi:10.1088/0963-0252/17/3/035016.

[2] V. Aubrecht, M. Bartlova, and O. Coufal. Radiative emission from air thermal plasmas with vapour of $\mathrm{Cu}$ or W. J. Phys. D: Appl. Phys., 43(43):434007, 2010. doi:10.1088/0022-3727/43/43/434007.

[3] S. Fesenko et al. Properties of thermal air plasma with admixing of copper and carbon. Journal of Physics:
Conference Series, 550(1):012008, 2014. doi:10.1088/1742-6596/550/1/012008.

[4] M. S. Benilov et al. Account of near-cathode sheath in numerical models of high-pressure arc discharges. $J$. Phys. D: Appl. Phys., 49(21):215201, 2016. doi: 10.1088/0022-3727/49/21/215201.

[5] O. Coufal. Composition and thermodynamic properties of thermal plasma up to $50 \mathrm{kK}$. J. Phys. D: Appl. Phys., 40(11):3371-3385, 2007. doi : 10.1088/0022-3727/40/11/018.

[6] OSC Technical Information Server. Opacity Project. http://opacities.osc.edu.

[7] R. Liebermann and J. Lowke. Radiation emission coefficients for sulphur hexafluoride arc plasmas. $J$. Quant. Spectrosc. Radiative Trans., 16(3):253-264, 1976. doi : 10.1016/0022-4073(76)90067-4.

[8] NIST. Atomic Spectra Database. https: //www.nist.gov/pml/atomic-spectra-database.

[9] R. L. Kurucz and B. Bell. Atomic Line Data. http://kurucz.harvard.edu/linelists.html.

[10] Southwest Research Institute. PHoto Ionization/Dissociation RATES. http://phidrates.space.swri.edu.

[11] G. Herzberg. Molecular Spectra and Molecular Structure. I. Spectra of Diatomic Molecules. New York: D. Van Nostrand Co., 1950.

[12] J. Lowke. Predictions of arc temperature profiles using approximate emission coefficients for radiation losses. J. Quant. Spectrosc. Radiative Trans., 14(2):111122, 1974. doi:10.1016/0022-4073(74)90004-1.

[13] V. Aubrecht and J. Lowke. Calculations of radiation transfer in $\mathrm{SF}_{6}$ plasma using the method of partial characteristics. J. Phys. D: Appl. Phys., 27(10):20662073, 1994. doi:10.1088/0022-3727/27/10/013.

[14] P. Kloc et al. Radiation transfer in air and air- $\mathrm{Cu}$ plasmas for two temperature profiles. J. Phys. D: Appl. Phys., 48(5):055208, 2015. doi: $10.1088 / 0022-3727 / 48 / 5 / 055208$.

[15] H. Nordborg and A. Iordanidis. Self-consistent radiation based modelling of electric arcs : I. Efficient radiation approximations. J. Phys. D: Appl. Phys., 41(13):135205, 2008 doi:10.1088/0022-3727/41/13/135205.

[16] P. Kloc, V. Aubrecht, and M. Bartlova. Numerically optimized band boundaries of Planck mean absorption coefficients in air plasma. J. Phys. D: Appl. Phys., 50(30):305201, 2017. doi:10.1088/1361-6463/aa7627.

[17] M. Modest. Radiative Heat Transfer. Oxford: Academic Press, 2013.

[18] M. Bartlova et al. Modelling of radiative transfer in air arc plasma. Plasma Physics and Technology, 4(3):261-264, 2017. doi:10.14311/ppt.2017.3.261.

[19] G. Raynal, P. J. Vergne, and A. Gleizes. Radiative transfer in $\mathrm{SF}_{6}$ and $\mathrm{SF}_{6}-\mathrm{Cu}$ arcs. J. Phys. D: Appl. Phys., 28(3):508-515, 1995. doi: 10.1088/0022-3727/28/3/010. 